(C) [2005] IEEE. Reprinted, with permission, from [S.W. Su, L. Wang, B.G. Celler, E. A, Estimation of Walking Energy Expenditure by Using Support Vector Regressions, Engineering in Medicine and Biology Society, 2005. IEEEEMBS 2005. 27th Annual International Conference of the, 2005]. This material is posted here with permission of the IEEE. Such permission of the IEEE does not in any way imply IEEE endorsement of any of the University of Technology, Sydney's products or services. Internal or personal use of this material is permitted. However, permission to reprint/republish this material for advertising or promotional purposes or for creating new collective works for resale or redistribution must be obtained from the IEEE by writing to pubs-permissions@ieee.org. By choosing to view this document, you agree to all provisions of the copyright laws protecting it 


\title{
Estimation of Walking Energy Expenditure by Using Support Vector Regression
}

\author{
S.W. Su†, L. Wangt, B.G. Celler†, E. Ambikairajah† and A.V. Savkin $\dagger$ \\ Human Performance Lab, School of Electrical Engineering \& Telecommunications \\ University of New South Wales,UNSW Sydney N.S.W. 2052 Australia \\ Email addresses: †(steven.su, b.celler, e.ambikairajah, a.savkin)@unsw.edu.au, †luwang@student.unsw.edu.au
}

\begin{abstract}
This paper develops a new predictor of walking energy expenditure from wireless measurements of body movements using triaxial accelerometers. Reliable data were collected from repeated walking experiments in different conditions on a treadmill with simultaneous measurement of expired oxygen and carbon dioxide. Support vector regression, a powerful non-linear regression method, was used to process and model the data. This novel processing method sets this investigation apart from existing papers. Good results were achieved in the robust estimation of walking related energy expenditure from a number of variables derived from triaxial accelerometer and treadmill speed .
\end{abstract}

\section{INTRODUCTION}

The main purpose of this paper is to examine the prediction of walking energy expenditure by using wireless piezo-resistive accelerometers. Energy expenditure is an important physiological parameter for the determination of functional health status, for the calculation of optimum insulin dosage for Type I diabetics and for many other clinical assessments in normal and pathological conditions. Triaxial accelerometers are relatively low cost, can be easily calibrated and have a more than adequate sensitivity and frequency response. They can be used to estimate energy expenditure using a number of simple linear regression $\operatorname{models}^{[1],[2],[3],[4],[5],[6],[7],[8],[9]}$.

The initial step in the application of linear regression is to set the significance test of linear relationship between inputs and outputs data. Normally, this requires the analysis of the correlation coefficient $(r)$ between variables. However, the correlation coefficient only measures the degree to which the data fit a linear relationship [10]. Even if a perfect mathematical non-linear relationship between two variables exists, the correlation coefficient may be small or even zero. This indicates that correlation analysis cannot be applied to modeling systems with strong non-linearity.

In paper [11], a non-linear model was used to estimate energy expenditure. In effect, better estimation results were achieved in comparison to the usage of linear models. However, two shortcomings have been identified in the paper. Firstly, no explanation was provided by the authors on their choice of model structure and it is well known that the choice of model structure is extremely crucial in modeling. Secondly, a non-linear optimization method was applied in the identification of parameters. This approach is non-convex and in order to achieve best fit with the available data, iterative methods are used that start with an initial guess of the unknown parameters. A global optimization result is often difficult to find.

Support vector regression (SVR) ${ }^{[12]}$ is an efficient nonlinear regression method. Complex non-linear relationships can be approximated by using the so called kernel transformation of SVR. Furthermore, SVR can be implemented by using efficient convex optimization. Thus, it is a very promising method for the modeling of non-linear processes. In this paper, we have applied the SVR method to find non-linear relationships of walking related variables and obtained several valuable results.

\section{EXPERIMENTS AND DATA PRE-PROCESSING}

The main aim of this study was to investigate energy expenditure (EEact) by analysing body acceleration. EEact can be calculated as total energy expenditure (EEtot) minus resting energy expenditure (REE). $\mathrm{O}_{2}$ consumption $\left(\mathrm{VO}_{2}\right)$ and $\mathrm{CO}_{2}$ production $\left(\mathrm{VCO}_{2}\right)$ are measured at 1-min intervals to calculate minute-by-minute EEtot by using the abbreviated Weir equation. The Harris Benedict equation is applied to calculate an individual's REE. Finally, EEact has been normalized for body weight $(\mathrm{W} / \mathrm{kg})$.

\section{A. Subjects}

Six young healthy male subjects volunteered to participate in the study. Their physical characteristics are presented in Table 1.

Table 1. Subject characteristics $(\mathrm{N}=6)$.

\begin{tabular}{|c|c|c|c|}
\hline & Mean & SD & Range \\
\hline Age (yr) & 31.61 & 5.78 & $23-37$ \\
\hline Height (cm) & 176.41 & 5.48 & $169-184$ \\
\hline $\begin{array}{c}\text { Body mass } \\
(\mathrm{kg})\end{array}$ & 74.31 & 9.35 & $60-85$ \\
\hline
\end{tabular}

B. Experimental Procedure

All experiments were conducted in the afternoon, and the subjects were permitted to have a light meal one hour before measurements were recorded. Initially, the subjects were asked to walk for about 10 minutes on the treadmill to familiarize themselves with the experiment. The subjects 
were then requested to walk at five levels of different speeds $(3,4,5,6$ and $7 \mathrm{~km} / \mathrm{h})$. Each level took a total period of 5 minutes, and was followed by a 10 -minute resting period. Throughout the experiments, continuous measurements of $\mathrm{O}_{2}$ consumption $\left(\mathrm{VO}_{2}\right), \mathrm{CO}_{2}$ production $\left(\mathrm{VCO}_{2}\right)$, (Moxus Metabolic Measurement System), and triaxial accelerometer outputs were made. Subjects' heart rates were also monitored during experiments.

\section{Acceleration measurement and analysis}

The wireless portable triaxial accelerometers that were used are piezoresistive accelerometers. They are particularly suitable for detection of human movement due to their sensitivity to very low frequencies. The triaxial accelerometer was attached to the lower back close to the subject's centre of gravity. Accelerations were measured in a body-fixed axis system with measurement directions in antero-posterior (x), medio-lateral (y), and vertical (z). Individual outputs from the three measurements are highpass $(0.11 \mathrm{~Hz})$ and low-pass $(20 \mathrm{~Hz})$ filtered outputs to suppress DC-response and high frequencies that cannot be expected to arise from human movement.

Filtered acceleration signals from the last 2-minute interval at the end of each walking stage were processed to produce various accelerometer output variables: $I A A_{x}, I A A_{y}$, $I A A_{z}, I A A_{\text {tot }}, H$, and $V$. It should be noted although the symbols: $I A A_{x}, I A A_{y}, I A A_{z}, I A A_{\text {tot }}$ are the same as in [1], they are slightly different from the definitions given in [1].

Specifically, $I A A_{x}, I A A_{y}, I A A_{z}$ are the average integrals of the absolute value of accelerometer output from $\mathrm{x}, \mathrm{y}, \mathrm{z}$ directions:

$$
\begin{aligned}
& I A A_{i}=\frac{1}{T} \int_{t=0}^{T}\left|a_{i}\right| d t, \quad i=x, y, z . \\
& I A A_{\text {tot }}=I A A_{x}+I A A_{y}+I A A_{z}
\end{aligned}
$$

The definitions for $\mathrm{H}$ and $\mathrm{V}$ are similar to the definitions provided in [11]. The $\mathrm{H}$ component is defined as the average integral of the square root of the sum of squared signals of the $\mathrm{x}$ and $\mathrm{y}$ axis. The $\mathrm{V}$ component is equal to the average integral of the absolute value of accelerometer output from $\mathrm{z}$ direction.

\section{RESULTS}

In this paper, we have utilised support vector regression to predict EEact by applying the information from a triaxial accelerometer or the speed of treadmill. In order to show the effectiveness of SVR, traditional linear regression is also performed. These two approaches are evaluated by the difference (Mean Square Error of estimation) between predicted and actual EEact under the following cases.

\section{A. Estimation of EEact based on IAA $A_{\text {tot }}$}

For the estimation of EEact by using a triaxial accelerometer, paper [9] reported that one of the best estimation of EEact can be achieved by using the summation of the (average) integrals of absolute accelerometer output in $\mathrm{x}, \mathrm{y}$, and $\mathrm{z}$ direction $\left(\mathrm{IAA}_{\mathrm{tot}}\right)$.

We applied both support vector regression and linear regression to investigate the relationship between EEact and IAA $_{\text {tot }}$ The regression results are shown in Figure 1-3. The regression error of SVR is 0.3714 . This is smaller than that of linear regression $(0.3935)$.

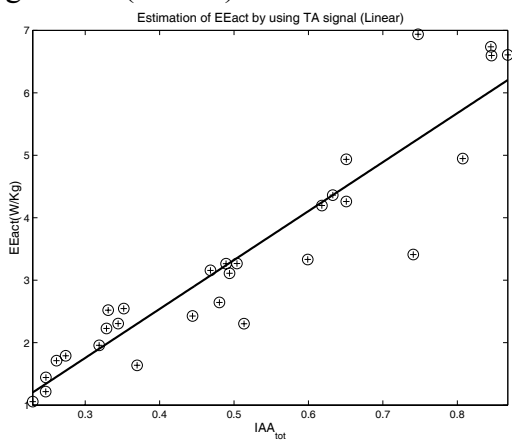

Fig. 1. Linear regression (EEact \& $\left.\mathrm{IAA}_{\mathrm{tot}}\right)$.

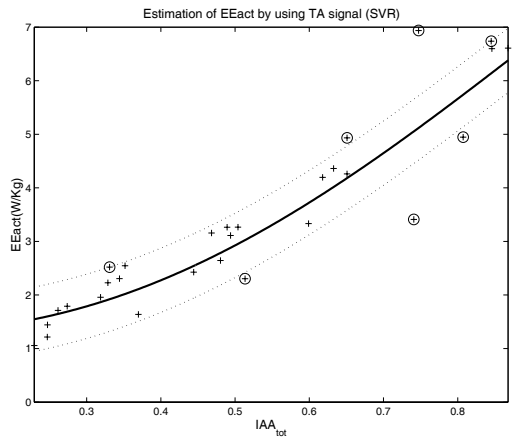

Fig. 2. Support vector machine regression (EEact \& $I_{A A}$ tot

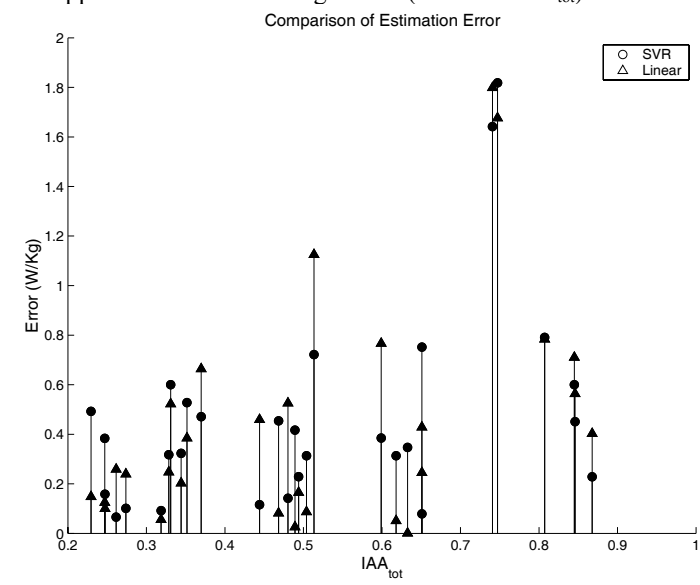

Fig. 3. Comparison of estimation errors between Linear regression and SVR 


\section{B. Estimation of EEact based on Hand V components}

In [11], Chen and Sun predicted EEact by using the vertical component $(\mathrm{V})$ and the horizontal component $(\mathrm{H})$. They established a multivariable linear model by applying multiply linear regression, and achieved good estimation results. This relationship deserved to be explored by using SVR. The regression results are presented in Figures 4-6. The regression error of SVR (0.3972) is much smaller than linear regression error $(0.4768)$ as shown in Fig. 6.

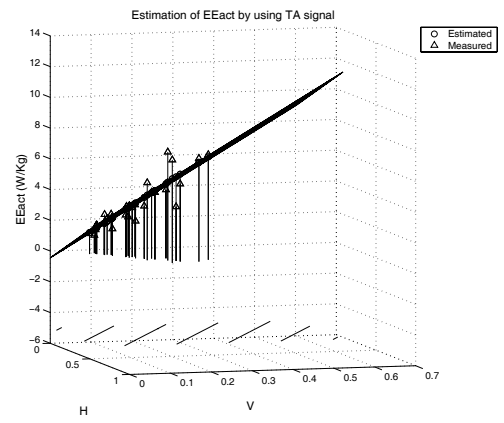

Fig. 4. Linear regression (EEact \& $\mathrm{H}$ andV)

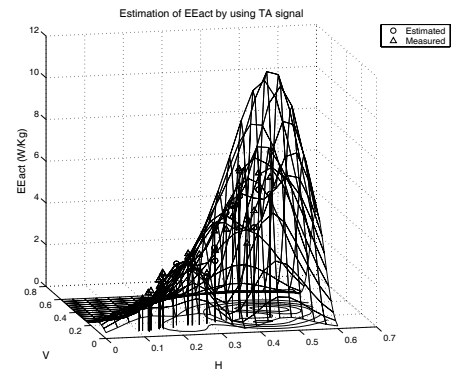

Fig. 5. SVR regression (EEact \& $\mathrm{H}$ and $\mathrm{V}$ )

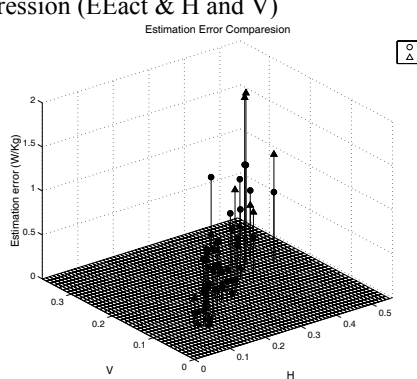

Fig. 6. Comparison of estimation errors (EEact \& $\mathrm{H}$ and V)

\section{Estimation of EEact based on $I A A_{x}$ and $I A A_{z}$}

Based on the analysis of triaxial accelerometer data, we found that two major acceleration components are in the $x$ and $z$ directions (the value of $a_{y}$ is smaller compared with $a_{x}$ and $\mathrm{a}_{\mathrm{z}}$.). We thus applied SVR and linear regression to predict EEact based on $\mathrm{IAA}_{\mathrm{x}}$ and $\mathrm{IAA}_{\mathrm{z}}$ only. Regression results are shown in Figures 7-9.

The regression error of SVR (0.3711) is much smaller than linear regression error $(0.5433)$, and it is nearly equal to the (SVR) estimation error $(0.3714)$ by using IAAtot, which required all three axial outputs.

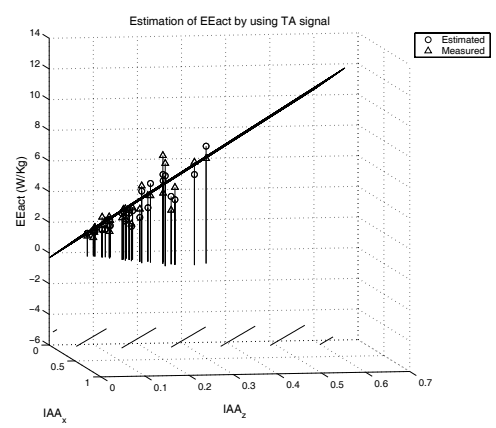

Fig. 7. Linear regression (EEact \& $\left.\mathrm{IAA}_{\mathrm{x}}, \mathrm{IAA}_{\mathrm{z}}\right)$

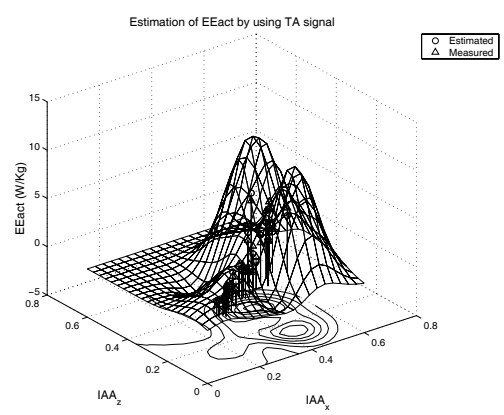

Fig. 8. SVR regression (EEact \& $\mathrm{IAA}_{\mathrm{x}}, \mathrm{IAA}_{\mathrm{z}}$ )

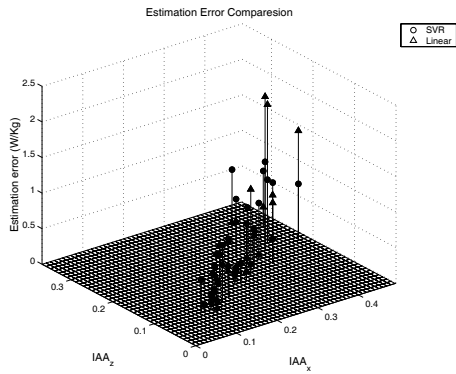

Fig. 9. Comparison of estimation errors $\left(\right.$ EEact \& $\left.\mathrm{IAA}_{\mathrm{x}}, \mathrm{IAA}_{\mathrm{z}}\right)$

\section{Estimation of EEact based on treadmill speed}

The relationship between $\mathrm{VO}_{2}$ (gross oxygen consumption) and treadmill speed has been reported. $\mathrm{VO}_{2}$ can be used as an measurement of $\mathrm{EE}_{\text {tot }}$. However, only approximate linear models have been provided in a given treadmill speed range. By using SVR, we establish a nonlinear model to estimate EEact $($ EEact $=$ EEtot-REE) by using the information of treadmill speed in all walking range (3-7 km/h). The regression results are shown in Figures 10 11. The estimation error of SVR (0.3367) is much smaller that that of linear regression (0.4747) as is shown in Fig. 11. Furthermore, it is the smallest estimation error of all cases studied. 


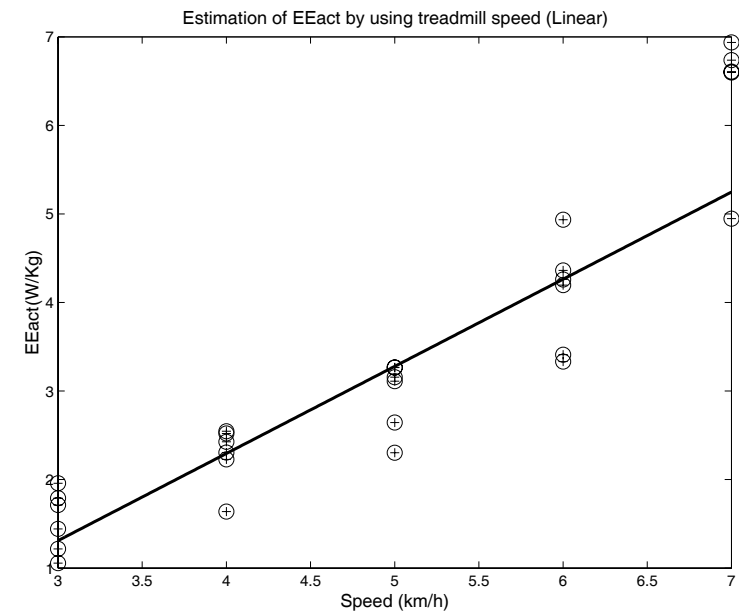

Fig. 10. Linear regression (EEact \& Speed)

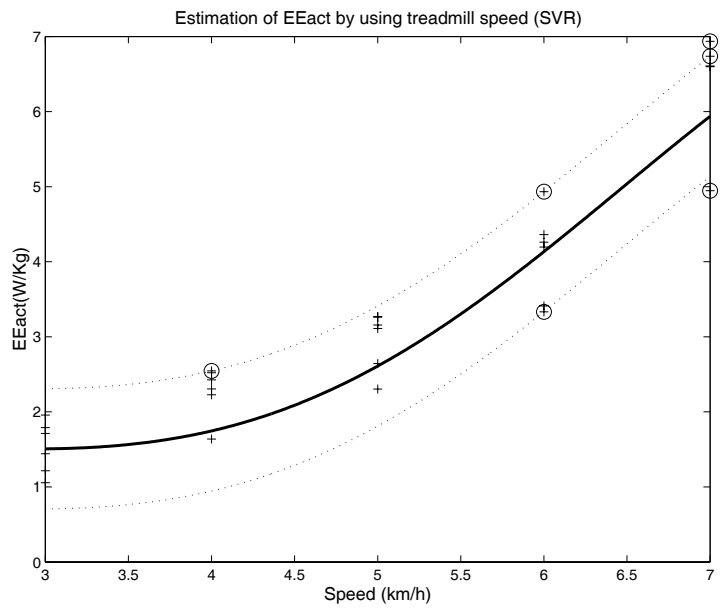

Fig. 11. SVR regression (EEact \& Speed)

Estimation errors (Mean Square Error) for both linear regression and SVR in all the experiments are summarized in Table 2.

Table 2. Estimation errors comparison (MSE).

\begin{tabular}{|c|c|c|c|c|}
\hline & $\begin{array}{c}\text { EEact } \\
\text { Vs } \\
\text { IAA }_{\text {tot }}\end{array}$ & $\begin{array}{c}\text { EEact Vs } \\
\mathrm{H} \& \mathrm{~V}\end{array}$ & $\begin{array}{c}\text { EEact } \\
\mathrm{Vs} \\
\mathrm{IAA}_{\mathrm{x}} \& \mathrm{I} \mathrm{AA}_{\mathrm{z}}\end{array}$ & $\begin{array}{c}\text { EEact Vs } \\
\text { treadmill } \\
\text { speed }\end{array}$ \\
\hline $\begin{array}{c}\text { Linear } \\
\text { Regreesion }\end{array}$ & 0.3935 & 0.4768 & 0.5433 & 0.4747 \\
\hline $\begin{array}{c}\text { Support } \\
\text { Vector } \\
\text { Regression }\end{array}$ & 0.3714 & 0.3972 & 0.3711 & 0.3367 \\
\hline
\end{tabular}

\section{CONCLUSION}

This paper estimates walking energy expenditure by using Support Vector Regression methods to model the relationship between data recorded from a wireless triaxial accelerometer and metabolic energy expenditure calculated from expired oxygen and carbon dioxide concentrations. Several useful results have been obtained:

- Non-linear models obtained by using SVR have lower estimation errors than linear models.

- $\quad$ Some relationships, which were described by using piece-wise linear models, can now be directly described by using a single non-linear model.

- Only two axes ( $\mathrm{x}$ and $\mathrm{z}$ ) are enough to achieve good estimate of EEact when the non-linear regression method SVR is applied.

This paper demonstrates that SVR is a powerful technique for the estimation of energy expenditure from accelerometer data.

\section{ACKNOWLEDGMENT}

The authors gratefully acknowledge the financial support of the Australian Research Council (Grant DP0452186).

\section{REFERENCES}

[1] Bouten, C. V., Sauren, A. A., Verduin, M. and Janssen, J. D. 1997, Effects of placement and orientation of body-fixed accelerometers on the assessment of energy expenditure during walking, Medical and Biological Engineering and Computing, 35, 50-56.

[2] Bouten, C. V., Koekkoek, K. T., Verduin, M., Kodde, R. and Janssen, J. D. 1997, A triaxial accelerometer and portable data processing unit for the assessment of daily physical activity, IEEE Transactions on Biomedical Engineering, 44, 136-147.

[3] J. Reswick, J. Perry, D. Antonelli, N. Su, and C. Freeborn, "Preliminary evaluation of the vertical acceleration gait analyzer (VAGA)," in Proc. 6th Annu. Symp. External Control Extremities, Dubrovnik, 1978, pp.305-314.

[4] G. Currie, D. Rafferty, G. Duncan, F. Bell, and A. L. Evans, "Measurement of gait by accelerometer and walkway: A comparison study," Med. Biol. Eng. Comput., vol.30, pp. 669-670, 1992.

[5] L. Brouha, "Evaluation of requirements of jobs," in Physiology in Industry. Oxford, U.K.: Pergamon, 1960, pp. 94-108.

[6] J. R. W. Morris, "Accelerometry-A technique for the measurement of human body movements," J. Biomech., vol. 6, pp. 729-736, 1973.

[7] A. H. Ismail, J. W. Baraby, and C. B. Smith, "Relationships between mechanical force and hysiological cost during gait in adult men," in Biomechanics: Proceeding of the C.I.C. Symposium Biomechanics, J. M. Cooper, Ed. Chicago: The Athlete Inst., 1971, pp. 99-106.

[8] J. D. Frost, "Triaxial vector accelerometry: A new method for quantifying tremor and ataxia," IEEE Trans. Biomed. Eng., vol. BME-25, pp.17-27, 1978.

[9] C. V. C. Bouten, K. R. Westerterp, M. Verduin, and J. D. Janssen, "Assessment of energy expenditure for physical activity using a triaxial accelerometer," Med. Sci. Sports Exerc., vol. 26, pp. 1516-1523, 1994.

[10] Bland, M. An Introduction to Medical statistics, third edition, Oxford University Press, 2000.

[11]Chen, KY and Sun, M. Improving energy expenditure estimation by using a triaxial accelerometer. Journal of applied Physiology, 83(6),21122122, 1997.

[12] H. Drucker, C. Burges, L.Kaufman, A. Smola, and V. Vapnik. Support vector regression machines. In M. Mozer, M. Jordan, and T. Petsche, editors, Advances in Neural Information Procession Systems 9, pages 155161, Cambridge, MA, 1997. 